\title{
REVISIÓN TAXONÓMICA DEL COMPLEJO AGAVE POTATORUM ZUCC. (AGAVACEAE): NUEVOS TAXA Y NEOTIPIFICACIÓN
}

\author{
Abisaí Josué García-Mendoza \\ Universidad Nacional Autónoma de México, Jardín Botánico, \\ Instituto de Biología, Apdo. postal 70-614, 04510 México, D.F. México. \\ abisai@ibunam2.ibiologia.unam.mx
}

\section{RESUMEN}

Se revisa el complejo taxonómico de Agave potatorum perteneciente al grupo Hiemiflorae. Se describen e ilustran dos taxa nuevos para la ciencia: Agave nussaviorum y A. nussaviorum subsp. deltoidea del estado de Oaxaca. La especie nueva se compara con Agave seemanniana Jacobi y A. potatorum Zucc. Se proporciona información sobre la distribución, usos, fenología y una clave para la separación de los taxa.

Palabras clave: Agavaceae, Agave nussaviorum, Agave potatorum, México, Oaxaca.

\begin{abstract}
The Agave potatorum complex, of group Hiemiflorae, is revised. Two new taxa from Oaxaca, Agave nussaviorum and A. nussaviorum subsp. deltoidea, are described and illustrated. The new species is compared with Agave seemanniana Jacobi and $A$. potatorum Zucc. Information concerning its distribution, ethnobotany, and phenology, and an identification key to the taxa of the complex are provided.
\end{abstract}

Key words: Agavaceae, Agave nussaviorum, Agave potatorum, Mexico, Oaxaca.

Durante la preparación del tratado taxonómico de Agave (Agavaceae) para la Flora del Valle de Tehuacán-Cuicatlán, se consideró necesario abordar la delimitación del complejo Agave potatorum. La especie pertenece al grupo Hiemiflorae 
(Gentry, 1982) caracterizado por presentar hojas ovadas a ampliamente lanceoladas, glaucas, con el margen dentado, inflorescencia paniculada a racemosa, flores agrupadas en umbelas, en ocasiones congestionadas, densamente bracteoladas, tépalos más largos que el tubo y filamentos insertados a la mitad o por arriba del tubo. Las plantas por lo general sin súrculos y reproducción preferentemente por semilla. El grupo Hiemiflorae contiene 13 especies que se distribuyen del sur de México (Puebla y Veracruz) a Nicaragua.

Agave potatorum fue descrita por Zuccarini en 1832, de plantas enviadas de México por Karwinski y cultivadas en el Jardín Botánico de Munich. Posteriormente, Zuccarini (1833) proporcionó información del hábito de crecimiento, medidas de la hoja, forma, tamaño de los dientes y ramificación de la inflorescencia (de acuerdo con Karwinski). No se conoce el área de procedencia de la especie; Zuccarini menciona "Crescit in imperio mexicano", aunque es altamente probable que haya sido colectada en las cercanías de Tehuacán, Puebla, pues fue publicada junto con otros dos agaves del mismo valle: Agave macroacantha Zucc. y A. karwinskii Zucc. Posteriormente se publicaron más nombres relacionados con la misma especie, algunos con ilustraciones, pero todos ellos basados en plantas cultivadas en diferentes jardines europeos. Gentry (1982) reunió bajo Agave potatorum a un número altamente variable de plantas que crecen en los estados de Oaxaca y Puebla; en consecuencia, al no encontrar caracteres morfológicos que se correlacionaran de manera consistente, aplicó un concepto amplio para la especie. El mencionado autor estudió poblaciones silvestres de Agave potatorum sensu lato, que colectó en 1952, 1963, 1965, 1967 y 1976, temporadas en las que herborizó 13 ejemplares con sus respectivos duplicados. Estas colectas, aunadas a otras 18 más, depositadas en los herbarios DES, ILL, MEXU, MICH, MO y US, no fueron suficientes para delimitar taxonómicamente las entidades observadas, lo que le impidió segregar taxa a nivel específico o subespecífico.

Después del trabajo de Gentry se realizaron numerosos recorridos de campo para observar las plantas en su hábitat, herborizar un mayor número de ejemplares y cultivar especímenes ex situ en el Jardín Botánico del Instituto de Biología de la Universidad Nacional Autónoma de México, para así evaluar su comportamiento y descartar una posible plasticidad morfológica. Finalmente, se llegó a la conclusión que se trata de tres especies diferentes. En el presente artículo se propone una nueva delimitación taxonómica de Agave potatorum; se describen e ilustran una nueva especie y una nueva subespecie para la ciencia y se considera necesario reubicar algunos especímenes de $A$. potatorum citados por Gentry (1982), ahora considerados dentro de A. nussaviorum o A. seemanniana. 
El tipo de Agave potatorum nunca fue elegido formalmente. Gentry (1982) menciona que en 1969 encontró dos especímenes en el herbario de Munich (M), que únicamente son parte de la inflorescencia. El más antiguo, "1867, Hortus Monacensis" representa una forma pequeña con ramas laterales cortas, semejante a la observada por el mencionado autor. El otro ejemplar "7.3.79, H.b.M." es de una inflorescencia más larga, con pedúnculo lateral de $8 \mathrm{~cm}$ de largo. Gentry considera que "Since these specimens date from 33 and 46 years after the publication of Zuccarini's species, their suitability as types is doubtful. However, they can be regarded as neotypes". Gentry no escogió de manera formal el neotipo, por lo que se prefirió proponer un nuevo tipo nomenclatural que respalde de manera adecuada el nombre.

Agave potatorum Zucc., Flora 15: 2 (Beiblatt 2): 96. 1832. Agave scolymus Karw. ex Salm-Dyck, Hort. Dyck.: 307. 1834. Agave elegans hort. ex Salm-Dyck, Bonplandia 7: 89. 1859. Agave latifolia hort. ex Salm-Dyck, Bonplandia 7: 89. 1859. Agave pulchra hort. ex Salm-Dyck, Bonplandia 7: 89. 1859. Agave saundersii Hook.f., Bot. Mag. 91. t. 5493. 1865. Agave verschaffeltii Lem. ex Jacobi, Hamburger GartenBlumenzeitung 21: 122. 1865. Agave scolymus var. polymorpha A. Terracc., Prim. Contr. Monogr. Agave. p. 38. 1885. Agave potatorum var. verschaffeltii (Lem. ex Jacobi) A.Berger, Die Agaven. p.186. 1915. Fig. 1.

Plantas perennes, solitarias, acaules, rara vez con 1-3 hijuelos estoloníferos; rosetas compactas, $30-50 \mathrm{~cm}$ alto, $40-60 \mathrm{~cm}$ diámetro. Hojas 30-60(-100) por planta, 15-35 cm largo, (5-)8-10 cm ancho, ovadas, oblongas o lanceoladas, suculentas, erectas, flexibles a rígidas, planas o algo cóncavas hacia el ápice, engrosadas hacia la base, glaucas a verde-glaucas; margen sinuado a crenado, mamilas 4-7(-10) mm alto; dientes de la parte media, 4-6(-12) mm largo, 4-6(-8) $\mathrm{mm}$ ancho, retrorsos, antrorsos o rectos, castaños a grisáceos, separados por $1-3 \mathrm{~cm}$; espina $3-4 \mathrm{~cm}$ largo, $0.4-0.7 \mathrm{~cm}$ ancho, recta o sinuosa, aguda, ampliamente acanalada a plana en el haz, decurrente por menos de $1 \mathrm{~cm}$, castaña a grisácea. Inflorescencia 3-5(-6) $\mathrm{m}$ alto, porción fértil en la mitad o tercio superior, paniculada, abierta, con (7-)11-25 ramas primarias 10$30 \mathrm{~cm}$ largo, separadas entre sí por $15-30 \mathrm{~cm}$, a veces con ramas secundarias $0.5-4$ cm largo; pedúnculo verde a pardo-rojizo, brácteas del pedúnculo $12-20 \mathrm{~cm}$ largo, 3.5-4 cm ancho en la base, triangulares, cartáceas, con algunos dientecillos en el margen, espina 0.5-2.5 cm largo, 0.7-1 cm ancho, pardo-rojiza; brácteas de las ramas primarias 5-8 cm largo, 2-5 cm ancho, ovadas, amplexicaules. Flores (15-)20-40 por umbela, (5-)5.5-7 cm largo, campanuladas a algo urceoladas, suculentas, verde- 


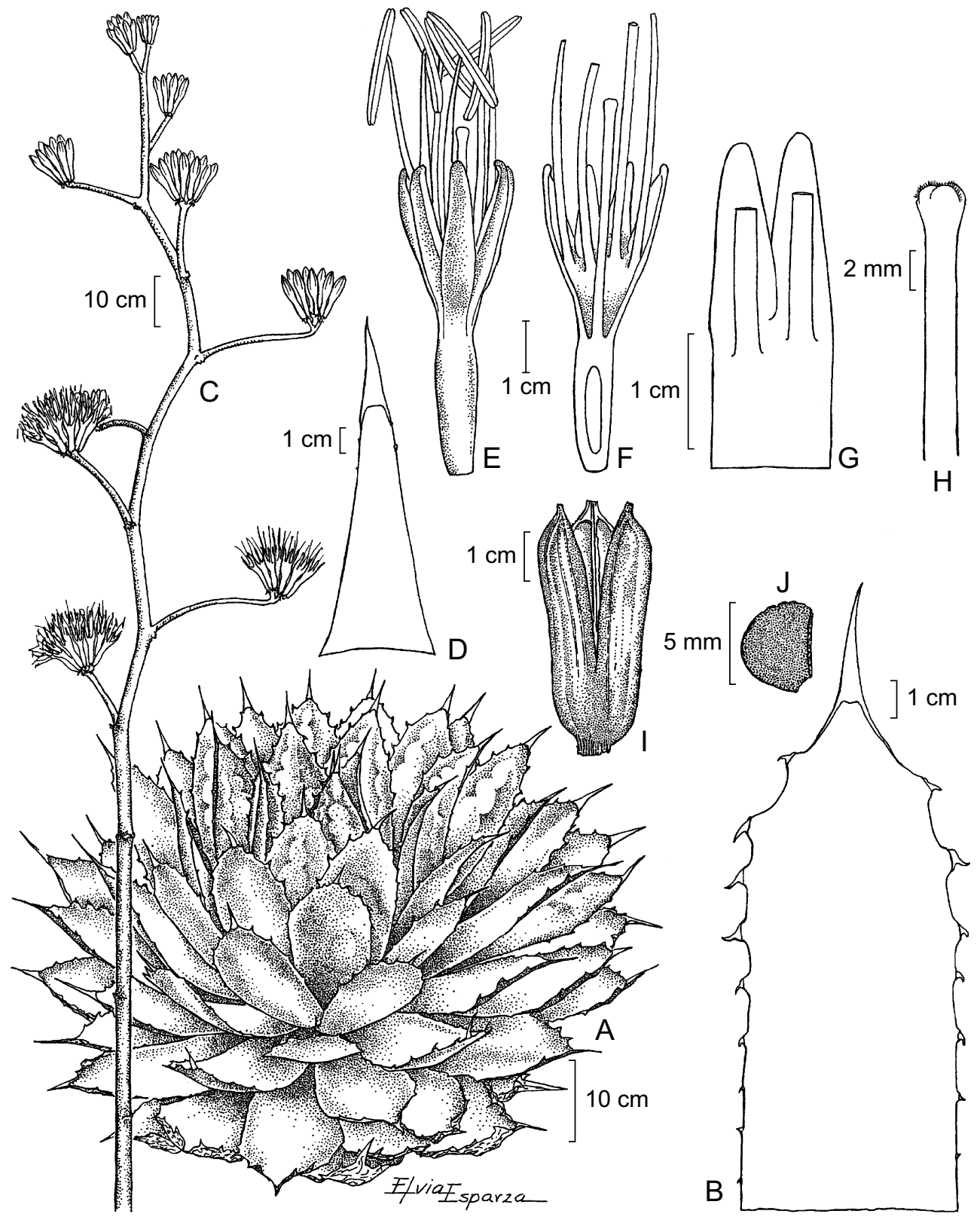

Fig. 1. Agave potatorum Zucc. A. roseta; B. hoja; C. inflorescencia; D. bráctea del pedúnculo; E. flor; F. flor disecada; G. detalle de la inserción del filamento; H. estilo y estigma; I. cápsula; J. semilla. 
amarillentas con tintes purpúreos o rojizos, ovario verde; pedicelos 1-1.5 cm largo; ovario 2.5-3 cm largo, 4-6 mm ancho, cilíndrico a ligeramente triquetro, cuello 2-3(-5) mm largo, tubo (1-)1.3-1.6 cm largo, (0.8-)1.3-1.8 cm diámetro en la porción distal, sulcado; tépalos 1.2-1.5(-2) cm largo, 3-6 mm ancho, triangulares u oblongos, erectos, gruesos, los externos 1-2 mm más largos que los internos, margen involuto, ápice cuculado, duro, los internos aquillados; filamentos 3-4(-4.5) cm largo, aplanados adaxialmente, insertados a la mitad o en el tercer cuarto superior del tubo, amarillentos, a veces con tintes purpúreos, anteras $1.5-2.5 \mathrm{~cm}$ largo, 2-3 $\mathrm{mm}$ ancho, rectas, versátiles, amarillentas; estilo 5.5-6 cm largo, estigma claviforme, trilobado. Cápsulas (3.5-) 4-6 cm largo, 1.5-2 cm ancho, oblongas, estipitadas, rostradas. Semillas 5-6 mm largo, 4-5 $\mathrm{mm}$ ancho, lunulares, con un ala apenas perceptible, aplanadas, negras.

Tipo. México, Puebla: 4-10 miles SW of Tehuacán along road to Zapotitlán, 5600-6000 feet (1700-1800 m), arid thorn forest over limestone hills, August 1963, H.S. Gentry, A. Barclay \& J. Argüelles 20427 (neotipo, aquí designado: MEXU!; isoneotipos: ARIZ!, US!).

Ejemplares examinados. México, OAXACA. Distrito Centro: cerro El Barrio (El Rosario) San Antonio, C. Conzatti et al. 4099 (MEXU, US); Monte Albán, A. Ramírez Laguna s.n. (MEXU); San Felipe, near City of Oaxaca, J.N. Rose y W. Hough 4592 (US). Distrito Coixtlahuaca: $7 \mathrm{~km}$ al SO del puente Calapa, municipio de Tepelmeme Villa de Morelos, A. García-Mendoza y E. Solano 7256, 7257 (MEXU, MO); $3 \mathrm{~km}$ al S de El Rodeo, A. Garcia-Mendoza et al. 7525 (MEXU); barranca del Otate, al SW de Hijadero Aria, P. Tenorio et al. 17103 (MEXU); cerro Maguey al W de San Antonio Abad, camino a La Mexicana, municipio de Santiago Ihuitlán Plumas, $P$. Tenorio 17539 (MEXU). Distrito Cuicatlán: 2 km en línea recta al NE de San Juan Tonaltepec, municipio de Santiago Nacaltepec, G. Juárez G. y C.A. Cruz 669 (MEXU); Barranca del río Chico, E de Cuicatlán, F. Miranda 4619 (MEXU). Distrito Etla: Tierra Larga, límites con San Juan del Estado, C.A. Cruz et al. 1815 (MEXU); $1 \mathrm{~km}$ al N de la desviación a Las Sedas, A. García-Mendoza 5704 (MEXU); misma localidad, cultivada en el Jardín Botánico, A. García-Mendoza 6105 (MEXU); 4.5 km O de El Parián camino a Nochixtlán, municipio de San Jerónimo Sosola, A. García-Mendoza y E. Solano 7307 (MEXU); $0.5 \mathrm{~km}$ al E de Las Sedas, D.H. Lorence et al. 4633 (MEXU). Distrito Huajuapan: $5 \mathrm{~km}$ adelante de Santiago Chazumba carretera a Tehuacán (km 58), A. Salinas 7644 (MEXU). Distrito Ixtlán: NE del río Natividad, municipio de Capulalpam de Méndez, S. Figue- 
roa B. y Y. Guzmán R. 766 (MEXU); $2 \mathrm{~km}$ al $\mathrm{S}$ de El Estudiante, A. García-Mendoza $y$ R. Torres 1959 (MEXU); 6-8 miles NE of Cd. Oaxaca along road to Ixtlán, H.S. Gentry 12057, 12079 (ARIZ, MEXU, US); Yavesía, I. Trejo 28 (MEXU). Distrito Miahuatlán: $12 \mathrm{~km}$ al S de Cuixtla, camino a Santa María Coatlán, A. GarcíaMendoza y L. de la Rosa 6359 (MEXU). Distrito Mixe: $6 \mathrm{~km}$ al N de Tamazulapan Mixes, camino a Totontepec, A. García-Mendoza et al. 1279 (MEXU). Distrito Tlacolula: $2 \mathrm{~km}$ al N de San Dionisio Ocotepec, A. García-Mendoza y R. Torres 1971 (MEXU); $10 \mathrm{~km}$ al N de Mitla, carretera a Ayutla Mixes, A. García-Mendoza y $R$. Torres 1973 (MEXU); $5 \mathrm{~km}$ al SE de Matatlán, carretera Mitla-Totontepec, cultivada en el Jardín Botánico, A. García-Mendoza 6554 (MEXU); 4 km al SO de Santa Rosa de Buenavista, municipio de San Sebastián Abasolo, A. García-Mendoza et al. 6815 (MEXU); cerro Nueve Puntas, aproximadamente $8 \mathrm{~km}$ al $\mathrm{N}$ de San Baltasar Guelavila, A. García-Mendoza y H. Espinosa 7426 (MEXU); monumento natural Yagul, A. García-Mendoza et al. 7595 (MEXU); 8 miles E of Mitla, H.S. Gentry 22344 (MEXU); al S de Santiago Matatlán, S.C. Solano y M.A. Vara 196 (ARIZ); $10 \mathrm{~km}$ al E de Santa María Albarradas, R. Torres y C. Martínez 12538 (MEXU); near Cueva Blanca, J. Schoenwetter JSOX-78 (US). Distrito Sola de Vega: $12.1 \mathrm{~km} \mathrm{~N}$ of Sola de Vega on the road to Oaxaca, B. Bartholomew et al. 3271 (MEXU). Distrito Zaachila: $3 \mathrm{~km}$ al $\mathrm{S}$ de San Cristóbal Cuatro Venados, camino a río Minas, $A$. GarcíaMendoza et al. 8192 (MEXU). Distrito Zimatlán: paraje La Cantera, A. Miranda y O. Hernández 738 (MEXU); cañada El Frijolón, $8 \mathrm{~km}$ al NE de La Cofradía, $A$. Miranda y O. Hernández 754 (MEXU). PUEBLA. A 7 km de San Andrés Cacaloapan, municipio Tepanco de López, I. Calzada 23189 (MEXU); $8.5 \mathrm{~km}$ pasando Azumbilla hacia La Esperanza, M. Cházaro y B.L. Mostul s.n. (MEXU); Puebla, A. Chimal 37 (MEXU); Tehuacan, Endlich 1909, 1929 a (MO); Cantera Tlayua, ca $2 \mathrm{~km}$ east to Tepeji, R. Felger 8520 (DES); $6 \mathrm{~km}$ al NO de Azumbilla carretera a Tehuacán, municipio de Chapulco, A. García-Mendoza y F. Palma 5629 (MEXU); $5 \mathrm{~km}$ al S de Tehuacán, carretera a Zapotitlán, cultivada en el Jardín Botánico, $A$. García-Mendoza 6121 (MEXU); La Virgen, 8 km al N de Zapotitlán, cultivada en el Jardín Botánico, A. García-Mendoza 6560 (MEXU); $13 \mathrm{~km}$ al S de Puerta Cañada, municipio de Cañada Morelos, A. García-Mendoza et al. 6664 (MEXU); $5 \mathrm{~km}$ al S de Puerto Las Palmas, municipio Izúcar de Matamoros, A. García-Mendoza et al. 6860 (MEXU); Loma Colorada, municipio Palmar de Bravo, A. García-Mendoza et al. 7090 (MEXU, MO); $10 \mathrm{~km}$ al S de Molcaxac, A. García-Mendoza et al. 7505 (MEXU); 22-24 km NE of Tehuacán, along highway to Veracruz, H.S. Gentry 12288 (ARIZ, MEXU, US); Tehuacan, D. Griffits s.n. (MO); paraje Agua Chiquita, en la cercanía del río Axamilpa, municipio Tepeji de Rodríguez, E. Guízar y G. Herrera 
4090 (MEXU); paraje Cuixosto, en la cercanía del río Axamilpa, municipio Tepeji de Rodríguez, E. Guizar y A. Miranda 4755 (MEXU); loma La Escobillera, a $1 \mathrm{~km}$ de Sabino Farol, municipio de Caltepec, E. Guizar y A. Miranda 4901 (MEXU); Izúcar de Matamoros, F. Miranda 2157 (MEXU); 10 miles S of Izúcar de Matamoros, along highway between Acatlán and Matamoros, E.C. Ogden y C.L. Gilly 51201 (ARIZ, MEXU, US); Tehuacán, elevaciones sierra de La Mesa, A. Ramírez Laguna s.n. (MEXU); near Tehuacan, J.N. Rose y R. Hay 5950 (US); J.N. Rose et al. 10024 (US); J.N. Rose y J.S. Rose 11261, 11269, 11421, 11426 (US); cerro Jabón, al W de La Mesa, municipio de Caltepec, P. Tenorio y C. Romero 4734 (MEXU, MO); cerro del muerto, al SW de San Simón, municipio de Caltepec, $P$. Tenorio y C. Romero 5052 (MEXU, MO); $10 \mathrm{~km}$ al NE de Azumbilla carretera a Esperanza, municipio de Chapulco, P. Tenorio 15200 (MEXU); Tehuacan, W. Trelease s.n. 12 ago 1903, 15 ago 1903, 5 feb 1905, 10 feb 1905 (MO); W of Tehuacan, W. Trelease s.n. (MO).

Fenología. Florece de fines de agosto a noviembre y fructifica de noviembre a marzo.

Hábitat y distribución. Agave potatorum se desarrolla en sitios planos o con poca pendiente, sobre suelos arenosos derivados de rocas calizas, en un intervalo altitudinal que va de los 1300 a $2400 \mathrm{~m}$, en climas áridos y semiáridos. Forma parte de la selva baja caducifolia, del matorral xerófilo con cactáceas columnares y del matorral xerófilo con Quercus. Su distribución abarca el extremo oriental de la cuenca del río Balsas, el Valle de Tehuacán-Cuicatlán, pie de monte de las sierras Mixtecas y montañas que rodean los Valles Centrales de Oaxaca.

Etimología: El epíteto específico proviene del latín potator, -oris bebedor (de vino).

Nombre común y usos: papalometl, papalomé, maguey papalomé, tobalá, jä näk tsääjts (mixe) (Vásquez, 2006). Se usa principalmente para la elaboración de mezcal, bebida alcohólica muy apreciada por sus cualidades organolépticas suaves. Las flores se emplean en la alimentación al igual que el pedúnculo floral cuando ha comenzado su desarrollo. En la medicina tradicional, las hojas se utilizan para disminuir hinchazones.

Agave potatorum se caracteriza por presentar rosetas compactas, con gran número de hojas planas con pequeñas mamilas y dientes; inflorescencia panicu- 
lada con ramas primarias de 10-30 cm de largo, separadas entre sí por 15-30 cm, presentes en la mitad o el tercio superior de la inflorescencia; brácteas más cortas que las ramas primarias; flores con el tubo campanulado a ligeramente urceolado, estambres insertados a la mitad del tubo o en el tercer cuarto superior, relación de tubo: tépalos 1:1 o incluso el tubo llega a ser hasta 2 veces más largo que los tépalos. En el extremo de los Valles Centrales de Oaxaca, en los distritos Mixe y Tlacolula al este, Miahuatlán y Sola de Vega al sur, Agave potatorum presenta una mayor variación en los órganos vegetativos y reproductivos; esto se debe seguramente a que florece sincrónicamente y es simpátrica con Agave seemanniana, especie con la que seguramente intercambia material genético. Estos especímenes con características morfológicas intermedias, de probable origen híbrido se enlistan a continuación.

Distrito Miahuatlán: $2 \mathrm{~km}$ al SO de San Jerónimo Coatlán, A. García-Mendoza et al. 4663 (MEXU); 7 to 8 miles SE of Miahuatlán along new road over Sierra Madre del Sur, H.S. Gentry 12106 (ARIZ, MEXU, US); $11.6 \mathrm{~km}$ al SO de San Pablo Coatlán, carretera Miahuatlán - Piedra Larga, R. Torres y A. Campos 10775 (MEXU, MO). Distrito Mixe: $4 \mathrm{~km}$ al E de Tlahuitoltepec, $3 \mathrm{~km}$ al N de Tamazulapan Mixes, A. García-Mendoza y R. Torres 2000 (MEXU). Distrito Sola de Vega: Yocúa, $4 \mathrm{~km}$ al N de Sola de Vega, A. García-Mendoza y H. Espinosa P. 7458, 7459 (MEXU). Distrito Tlacolula: $10 \mathrm{~km}$ al S de Cuajimoloyas, A. García-Mendoza 5672 (MEXU); 8 km al N de Díaz Ordaz, A. García-Mendoza et al. 5782 (MEXU); misma localidad, cultivada en el Jardín Botánico, A. García-Mendoza 6317, 6559 (MEXU); $9 \mathrm{~km}$ al S de Matatlán, en la base del cerro Nueve Puntas, A. GarcíaMendoza y H. Espinosa 7429 (MEXU); 8 miles E of Mitla along road to Ayutla, H.S. Gentry 22344 (MEXU).

Agave nussaviorum García-Mend. Fig. 2.

Ex affinitate $A$. potatorum Zucc. et $A$. seemanniana Jacobi, ab A. potatorum differt inflorescentia racemosa congesta, umbellis subsessilibus inter se proximissimis, paucifloris, floribus $1 / 3$ ad $1 / 6$ partis apicalis occupantibus; bracteis quam ramis longioribus; rosulis apertis laxis, foliis minus numerosis; margine mamillis magnis instructo, dentibus in mamillarum apicibus decurrentibus, floribus brevitubulatis, longitudine tubi quoad rationes longitudinis tepalorum 1:1.5-2.5; ab A. seemanniana differt partibus vegetativis reproductivisque magnitudine minori, foliis magis numerosis, lanceolatis vel oblongis et inflorescentia racemosa. 
Plantas perennes, solitarias, acaules; rosetas abiertas a compactas, $30-50 \mathrm{~cm}$ alto, 40-60 cm diámetro. Hojas 25-40 por planta, (12-)20-40 cm largo, (5-)7-11 cm ancho, lanceoladas, oblongas a ovadas, suculentas, erectas a recurvadas, flexibles, algo cóncavas hacia la parte media y ápice, verde-amarillentas, glaucas hacia la base; margen crenado, mamilas prominentes hacia la parte media de la lámina, 5-10 mm alto, variadamente curvadas; dientes (5-)8-12 mm largo, (5-) 8-10 mm ancho, con base amplia que cubre la cúspide de la mamila, retrorsos, antrorsos o rectos, castaños a grisáceos, separados por 2-3.5 cm, en ocasiones con dientecillos intersticiales, hacia la base de la hoja los dientes son pequeños y más cercanos entre sí; espina 2-3(-4) cm largo, 0.3-0.5(-0.8) cm ancho, sinuosa a recta, acanalada en el haz, decurrente por 1-2 $\mathrm{cm}$, castaña a grisácea. Inflorescencia (1.5-)3-5(-6) m alto, porción fértil en 1/3 a 1/6 de la parte distal, racemosa, congesta, flores en 20-40(-60) umbelas, ramas de menos de $3 \mathrm{~cm}$ de largo, separadas entre sí por $3-5 \mathrm{~cm}$; pedúnculo verdoso a púrpura; brácteas del pedúnculo $12-17 \mathrm{~cm}$ largo, $3.5-4.5 \mathrm{~cm}$ ancho en la base, triangulares, cartáceas, con algunos dientecillos en el margen, espina 2-3.5 cm largo, 0.3-0.5(-1) $\mathrm{cm}$ ancho, pardo-rojiza; brácteas de las umbelas 2.5-3.5(-5) cm largo, 2-3.5 cm ancho, ovadas, cartáceas, amplexicaules, más largas que las ramas, espina de menos de $5 \mathrm{~mm}$. Flores 6-12 por umbela, 4.5-5.5(-7) cm largo, ligeramente campanuladas, suculentas, amarillentas con tintes purpúreos, ovario verde; pedicelos 2-5(-10) mm largo; ovario 2.3-3(-3.5) cm largo, 4-6 mm de ancho, cilíndrico, cuello 2-3(-5) mm largo, tubo 0.7-1(-1.3) $\mathrm{cm}$ largo, 0.7-1 cm diámetro en la porción distal, ligeramente sulcado; tépalos 1.5-2.5 $\mathrm{cm}$ largo, 2-5 $\mathrm{mm}$ ancho, triangulares u oblongos, gruesos, los externos ligeramente más largos que los internos, margen involuto, ápice cuculado, duro, los internos aquillados; filamentos (2.5-)3.5-4.5(-5.5) cm largo, insertados 2-3 $\mathrm{mm}$ por debajo de la base de los tépalos, amarillentos con tintes purpúreos, anteras 2-2.5 $\mathrm{cm}$ largo, $2 \mathrm{~mm}$ ancho, versátiles, amarillentas; estilo 4-6 cm largo, estigma claviforme, trilobado. Cápsulas 4-6 cm largo, 2-2.5 cm ancho, oblongas a ovadas, estipitadas, rostradas. Semillas 5-6 $\mathrm{mm}$ largo, 4-5 $\mathrm{mm}$ ancho, lunulares, con un ala muy angosta, aplanadas, negras.

Tipo. México, Oaxaca: distrito Nochixtlán, municipio de Santo Domingo Yanhuitlán, $1 \mathrm{~km}$ al NO de Yanhuitlán, $2238 \mathrm{~m}$, zona erosionada en el bosque de Quercus, 5 septiembre 2008, A. García-Mendoza, S. Franco y F. Martínez 9204 (holotipo: MEXU; isotipos: ENCB, IEB, MO, OAX, US).

Paratipos. México, OAXACA. Distrito Coixtlahuaca: km 195.5 carr. Oaxaca - Tehuacán, cerca de Monte Verde, municipio Santa María Nativitas, A. García-Mendoza et al. 9206 (ENCB, MEXU); Distrito Huajuapan: palmetum, H. Bravo Hollis 


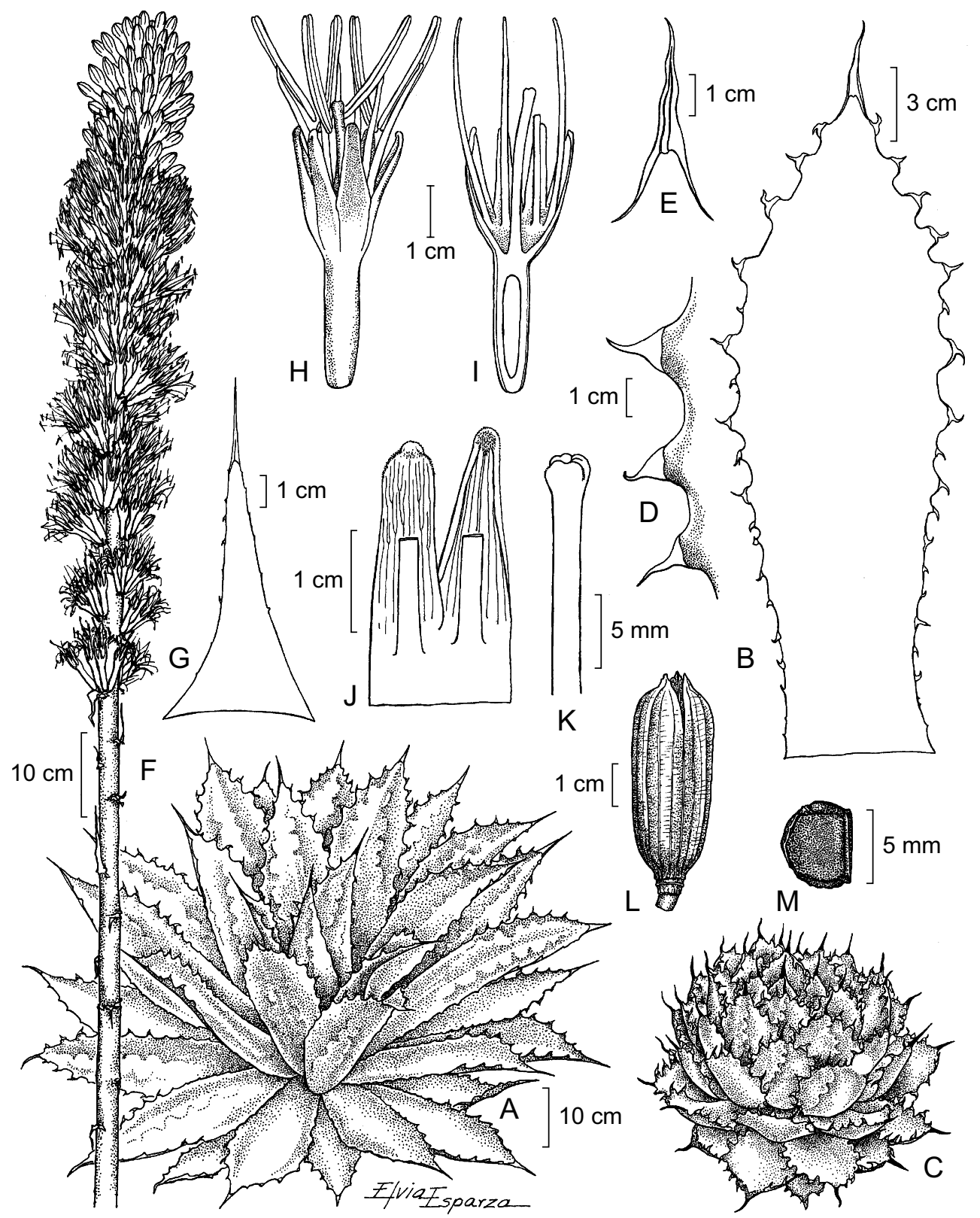

Fig. 2. Agave nussaviorum subsp. nussaviorum García-Mend. A. roseta; B. hoja; C. roseta de forma compacta; D y E. detalle de mamilas del margen y espina; F. inflorescencia; G. bráctea del pedúnculo; H. flor; I. flor disecada; J. detalle de la inserción del filamento y ápice de tépalos; K. estilo y estigma; L. cápsula; M. semilla. 
s.n. (MEXU); $10 \mathrm{~km}$ al NO de Huajuapan, carretera a Acatlán, A. García-Mendoza et al. 3628 (MEXU); El Mirador, $9 \mathrm{~km}$ al NO de Huajuapan, A. García-Mendoza y J. Reyes 4933 (MEXU); cañada de Lencho, $6 \mathrm{~km}$ al SO de San Marcos Arteaga, carretera Huajuapan - Tonalá, A. García-Mendoza y F. Palma Cruz 5804 (MEXU); misma localidad, cultivada en el Jardín Botánico, A. García-Mendoza 6118 (MEXU); $6 \mathrm{~km}$ al SE de Huajuapan carretera a Tamazulapan, A. García-Mendoza et al. 6328 (MEXU); $1 \mathrm{~km}$ al NO sobre la desviación a Guadalupe Cuautepec, A. García-Mendoza et al. 7096 (MEXU, MO); a $1 \mathrm{~km}$ sobre la desviación a Guadalupe Cuautepec, A. García-Mendoza y E. Solano 7336 (MEXU, MO); 6 miles NW of Huajuapan along highway, H.S. Gentry 22401 (ARIZ, MEXU, US); ca. 2 miles N of Huajuapan along highway to Puebla, H.S. Gentry y M. Gentry 23656 (ARIZ, DES, US); Paraje Palo Flor, $4 \mathrm{~km}$ al NO de Santo Domingo Yodohino, municipio Santo Domingo Yodohino, J. López Moreno 279 (MEXU); Huajuapan, km 80, H. Quero s.n. (MEXU); rancho La Cruz, $31 \mathrm{~km}$ al O de Huajuapan, $R$. Torres et al. 14162 (MEXU). Distrito Juxtlahuaca: a $4.5 \mathrm{~km}$ de San Sebastián Tecomaxtlahuaca, senda para río Seco y arroyo Timbre, J.I. Calzada 21808 (MEXU); lomas de Yucha Cuanshii, municipio de San Juan Mixtepec, J. Reyes 1227 (MEXU). Distrito Nochixtlán: cañada Yucuduza, municipio de Asunción Nochixtlán, D. Aquino García 201 (MEXU); $12 \mathrm{~km}$ al E de Nochixtlán y $1 \mathrm{~km}$ al $\mathrm{N}$ de río Salinas, A. García-Mendoza y L. de la Rosa 6175 (MEXU); about 2 miles NW of Yanhuitlán along highway, H.S. Gentry 12111 (ARIZ, MEXU, US); 1 mile NW of Yanhuitlán along highway, H.S. Gentry 22399 (ARIZ, MEXU, US); below Pan-Am. Hwy. at Puerto Herrera (km 420) just NW of Yanhuitlán, H.H. Iltis et al. 1147 (ENCB); $21 \mathrm{~km}$ von Llano Verde, desv. a Jaltepec Morelos, B. Leuenberger y C. Schiers 2773 (MEXU); 1 mile north of La Joya, along highway from Cd. Oaxaca to Acatlán, E.C. Ogden y C.L. Gilly 51193 (MEXU, US); Toxi, F. Palma Cruz 13 (MEXU). Distrito Teposcolula: $5.3 \mathrm{~km}$ del entronque del puente río del Oro sobre el acueducto de la CFE, carretera Tamazulapan - Huajuapan, J.I. Calzada 23786 (MEXU); $7 \mathrm{~km}$ al SO de San Andrés Lagunas camino a Teposcolula, A. García-Mendoza 893 (MEXU); alrededores de Anama, $3 \mathrm{~km}$ al S de San Vicente Nuñú, municipio de San Vicente Nuñú, A. García-Mendoza et al. 5250; $6 \mathrm{~km}$ al O de Teposcolula camino a San Pedro Yucunama, A. García-Mendoza 8837 (MEXU); about $15 \mathrm{mi}$ NW of Tejupan, H.S. Gentry 12124 (MEXU). Distrito Tlaxiaco: $3 \mathrm{~km}$ al NO de Tlaxiaco y a $3 \mathrm{~km}$ de la desviación a Santiago Nundiche, municipio de Santiago Nundiche, A. Castañeda et al. 95 (MEXU).

Fenología. Florece de fines de agosto a diciembre y fructifica de noviembre a marzo. 
Hábitat y distribución. Agave nussaviorum se desarrolla en las montañas de la Mixteca Alta, en sitios planos o laderas de los cerros, sobre suelos derivados de rocas calizas o areniscas, en un intervalo altitudinal de los 1700 a $2500 \mathrm{~m}$. Forma parte del bosque de Quercus-Pinus con Juniperus y matorral esclerófilo con Rhus, Brahea, Arctostaphylos, Nolina, Comarostaphylis, etc. Su distribución se restringe a las partes altas de las montañas en los distritos de Coixtlahuaca, Huajuapan, Juxtlahuaca, Nochixtlán, Teposcolula y Tlaxiaco.

Etimología. El epíteto específico alude a la región Mixteca Alta oaxaqueña y a sus pobladores ( $\tilde{n} u u$ savi $=$ tierra de nubes o habitantes del pueblo de las nubes), área de distribución geográfica de la especie.

Nombre comunes y usos. Papalometl, papalomé, "yavi ticushi” (mixteco). Se utiliza principalmente como planta alimenticia y medicinal. Los mixtecos elaboran el "yahui ndodo", alimento de sabor agridulce muy apreciado en épocas pasadas; asimismo, el quiote joven se cuece en horno y se come como golosina; las flores (cacayas o tibilos) se consumen en guisos y también cocidas se mezclan con masa de maíz para hacer tortillas. Al jugo de las hojas se le atribuyen propiedades medicinales para combatir enfermedades del sistema circulatorio como coágulos sanguíneos y diabetes. Las hojas tatemadas (semicocidas al fuego) se emplean para aliviar inflamaciones provocadas por golpes o caídas en los animales y el hombre. López (2008) menciona que tiene hasta siete usos diferentes, entre otros, la inflorescencia se emplea como forraje, el quiote seco en la construcción y como combustible.

Agave nussaviorum se diferencia de A. potatorum por el tipo de inflorescencia racemosa con ramas de las umbelas muy cortas, muy cercanas entre sí y con pocas flores, la parte fértil cubre de 1/3 a 1/6 de la parte distal de la inflorescencia, siendo las brácteas más largas que las ramas. Las rosetas tienen menor número de hojas, son más abiertas y de coloración verdoso-amarillenta, las hojas, por lo general, tienen mamilas grandes y dientes decurrentes sobre el ápice de la mamila, en ocasiones se observan dientecillos intersticiales. Las flores son subsésiles con tubos cortos, en una relación de 1:1.5-2.5 con respecto a los tépalos; los filamentos insertados 2-3 mm por debajo de la base de los tépalos. El hábitat corresponde al clima templado, es fresco y húmedo, con bosque de Quercus-Pinus, bosque de Pinus y matorral esclerófilo. En algunos sitios erosionados y con suelos pobres, las rosetas son globosas y pequeñas, con inflorescencias no mayores a $2 \mathrm{~m}$ de alto. 
De Agave seemanniana se diferencia porque esta especie tiene todos sus órganos vegetativos y reproductivos más grandes, tiene menos hojas, de forma espatulada o ampliamente lanceolada, de color glauco, recurvadas, margen de la hoja sinuoso, dientes grandes, mayormente separados entre sí e inflorescencia de un tamaño mayor, con ramas primarias, secundarias e incluso terciarias. Agave nussaviorum coexiste con $A$. convallis, A. atrovirens, A. salmiana y A. angustifolia (en pocos sitios) y hacia el norte con $A$. potatorum.

Es probable que Agave nussaviorum tenga un origen híbrido entre A. potatorum (la especie morfológicamente más parecida) y alguna especie del subgénero Littaea, como A. convallis o A. angustiarum; sin embargo, no hay evidencias que sustenten dicho proceso. Actualmente, A. nussaviorum ocupa un área geográfica contigua a la de $A$. potatorum, crece en un hábitat diferente, donde se reproduce de manera sexual exitosa. En este posible proceso habría que considerar también el manejo humano, puesto que, son especies ampliamente utilizadas, aunque con fines distintos. Algunas evidencias morfológicas que sugieren una hibridación se presentan en los siguientes ejemplares, recolectados en áreas perturbadas por actividades humanas.

Distrito Ixtlán: ladera sobre el Río Grande, al E de Yahuiche, A. GarcíaMendoza y F. Martínez 7055 (MEXU). Distrito Nochixtlán: Arroyo Tinú, $10 \mathrm{~km}$ al SE de Nochixtlán, A. García-Mendoza y E. Solano 7300 (MEXU). Distrito Teposcolula: El Organito, $1 \mathrm{~km}$ al N de Rancho Peñasco, San Pedro Nopala, A. GarcíaMendoza y L. de la Rosa 6158 (MEXU).

La información anterior corresponde a la subespecie típica $A$. nussaviorum ssp. nussaviorum, pues a continuación se propone como nueva y se describe:

Agave nussaviorum García-Mend. subsp. deltoidea García-Mend., subsp. nov. Fig. 3.

A subspecie typica differt foliis deltoideis longioribus latioribusque, margine corneo continuo vel denticulis quam $5 \mathrm{~mm}$ brevioribus margine adpressis, et spina 6-10 cm longa, longe decurrenti.

Plantas perennes, solitarias, acaules; rosetas abiertas, $50-70 \mathrm{~cm}$ alto, $60-80 \mathrm{~cm}$ diámetro. Hojas 20-40 por planta, 20-40 cm largo, (8-)14-20 cm ancho en la parte basal, deltoides a ampliamente lanceoladas, suculentas, rígidas, aplanadas o cóncavas, verde-amarillentas, en ocasiones zonadas con bandas transversales glaucas; margen entero, recto y córneo o escasamente dentado; dientes (1-)4-5 mm largo, sobre una 
base amplia (0.6-)1-1.3 cm ancho, retrorsos, adpresos al margen o rara vez libres y recurvados, grisáceos, separados por 1-2 cm; espina (4-)6-10 cm largo, 0.5-0.8 cm ancho, decurrente por 10-14 cm o continua con el margen, acanalada en el dorso, grisácea. Inflorescencia 4.5-6.5 m alto, pedúnculo 3-5 m, verdoso, porción fértil en el último tercio superior, racemosa, congesta, flores aglomeradas en 30-60 umbelas subsésiles de 1.5-2.5 cm largo, separadas entre sí por 3-5 cm; brácteas del pedúnculo de 12-18 cm largo, 2-5 cm ancho en la base, triangulares, con algunos dientecillos en el margen, cartáceas, espina (2-)3-4 cm largo, (0.3-)0.6-1 cm ancho, pardo-rojiza, brácteas de las ramas $4 \mathrm{~cm}$ largo, $3 \mathrm{~cm}$ ancho, ovadas, más largas que las ramas. Flores 4-10 por umbela, 4.5-6(-7) cm largo, algo campanuladas, verde-amarillentas, ovario verde; subsésiles con pedicelos hasta de $5 \mathrm{~mm}$ largo; ovario $2.5-2.8 \mathrm{~cm}$ largo, 4-8 $\mathrm{mm}$ ancho, cilíndrico, cuello 3-5 $\mathrm{mm}$ largo, tubo $1-1.5 \mathrm{~cm}$ diámetro, $1-1.3 \mathrm{~cm}$ ancho en la porción distal; tépalos (1.5-)2.5-2.8 cm largo, 3-8 mm ancho, triangulares u oblongos, los externos con margen involuto, ápice cuculado, duro, los internos aquillados; filamentos (3.5-)4-5 cm largo, insertados 2-4 $\mathrm{mm}$ por debajo de la base de los tépalos, amarillentos, anteras 1.5-2.5 cm largo, 2-3 mm ancho, versátiles, amarillentas; estilo 5-5.5 cm largo, estigma trilobado. Cápsulas 3.5-4 cm largo, 1.5-2 $\mathrm{cm}$ ancho, oblongas, rostradas. Semillas 5-6 $\mathrm{mm}$ largo, 3-4 $\mathrm{mm}$ ancho, lunulares, con un ala muy angosta, aplanadas, negras.

Tipo. México, Oaxaca: distrito Teposcolula, municipio de San Pedro Nopala, cerro la Peña del Tigre, $10 \mathrm{~km}$ al $\mathrm{N}$ de Nopala, $2600 \mathrm{~m}$, matorral rosetófilo, 30 abril 1995, A. García-Mendoza y F. Martínez 6051 (holotipo: MEXU; isotipo: OAX).

Paratipos. México, OAXACA. Distrito Teposcolula: cerro Pericón, $5 \mathrm{~km}$ al NO de Nopala, municipio de San Pedro Nopala, A. García-Mendoza et al. 3959 (MEXU); cerro La Peña del Tigre, $10 \mathrm{~km}$ al N de Nopala, A. García-Mendoza y F. Martínez 6057 (MEXU); misma localidad, cultivada en el Jardín Botánico, $A$. García-Mendoza 7237 (MEXU); Cerro Malintzin, $8 \mathrm{~km}$ al N de Nopala, A. GarcíaMendoza et al. 6689 (MEXU).

Fenología. Florece de septiembre a octubre y fructifica de diciembre a abril.

Hábitat y distribución. Agave nussaviorum var. deltoidea se desarrolla en sitios pedregosos sobre suelos derivados de rocas ígneas, en altitudes de 2400 a 2600 m; forma parte del matorral rosetófilo dominado por Agave convallis, Agave nussa- 
García-Mendoza: Revisión taxonómica del complejo Agave potatorum (Agavaceae)

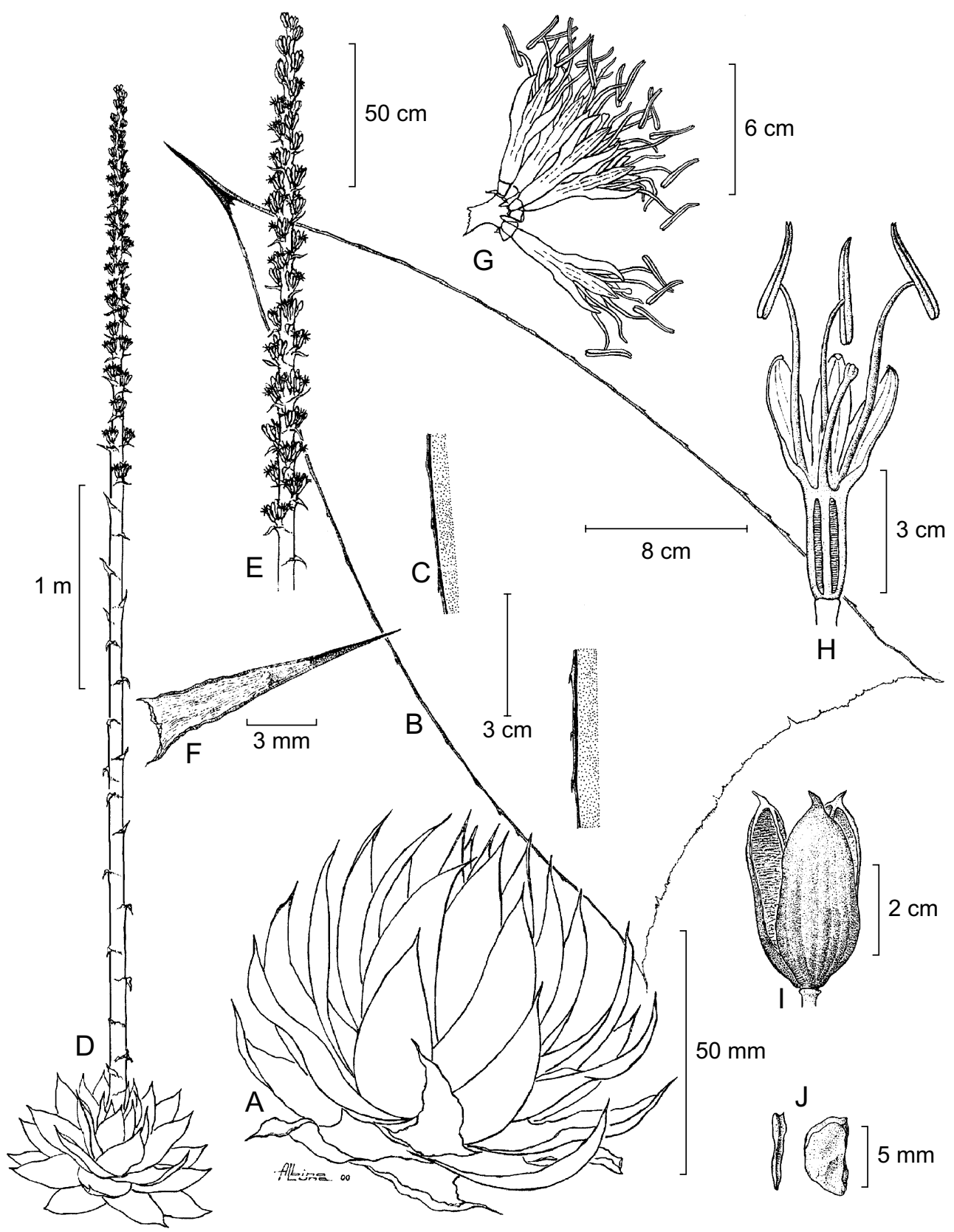

Fig. 3. Agave nussaviorum subsp. deltoidea García-Mend. A. roseta; B. hoja; C. detalle del margen; D. planta en floración; E. inflorescencia; F. bráctea del pedúnculo; G. umbela; H. flor disecada; I. cápsula; J. semilla. 
viorum, Dasylirion serratifolium, Furcraea longaeva, Hechtia sp., Nolina sp. y Yucca mixtecana. Aparentemente es endémica de la localidad tipo y áreas cercanas.

Etimología. El epíteto específico alude a la forma de las hojas.

La subespecie deltoidea se diferencia de la subespecie típica por las hojas deltoides más largas y anchas, con margen córneo continuo o con dientecillos menores a $5 \mathrm{~mm}$ de largo, adpresos al margen, espina de 6 a $10 \mathrm{~cm}$ de largo, largamente decurrente. Se desarrolla hacia el este del área de distribución de la subespecie típica, en un hábitat diferente, a mayor altitud y sobre suelos derivados de roca volcánica, en donde es escasa y se restringe a sustratos de origen ígneo.

Agave seemanniana Jacobi, Abh. Schles. Ges. Vaterl. Cult., Abth. Naturwiss. 1868: 154. 1869, Agave scolymus var. seemanniana (Jacobi) A. Terracc., Prim. Contr. Monogr. Agave. p. 38. 1885. Agave caroli-schmidtii A. Berger, Die Agaven. p. 199. 1915. Agave guatemalensis A. Berger, Die Agaven. p. 201. 1915. Agave seemanniana var. perscabra Trel., Trans. Acad. Sci. St. Louis 23: 137. 1915. Agave tortispina Trel., Trans. Acad. Sci. St. Louis 23: 135. 1915. Agave pygmaea Gentry, Agaves Cont. N. Amer. p. 494. 1982. Agave seemanniana subsp. pygmaea (Gentry) B. Ullrich, Brit. Cactus Succ. J. 10: 27. 1992. Figs. 4D, 5D; Gentry, 1982: 496, fig. 17.23.

Plantas perennes, solitarias, acaules; rosetas abiertas, (0.8-)1-1.5 m alto, (1.3-)1.5-2 m diámetro. Hojas (16-)30-40 por planta, 40-55 cm largo, (10-)14-22 cm ancho, espatuladas, ampliamente lanceoladas u ovadas, suculentas, recurvadas a erectas, angostas hacia la base, glaucas, verde-glaucas o verde-amarillentas, zonadas; margen crenado, mamilas (0.7-)1-1.5(-2) cm alto; dientes (0.6-)1-1.5 cm largo, 1-1.4 cm ancho, con base amplia que cubre la cima de la mamila, retrorsos a rectos, pardos a grisáceos, separados por (3-)4-5.5 cm; espina (3-)4.5-5 cm largo, 0.5-1 $\mathrm{cm}$ ancho, sinuosa, decurrente por $2-6 \mathrm{~cm}$, ampliamente acanalada en el haz. Inflorescencia paniculada, 4-6(-7) $\mathrm{m}$ alto, abierta, porción fértil en la mitad superior, silueta oblonga, flores en 15-30 umbelas laterales, ramas primarias $15-30(-50) \mathrm{cm}$ largo, separadas entre sí por 15-30 cm, ramas secundarias (1-)3-7(-10) cm, ramas terciarias ocasionalmente presentes $1-1.5 \mathrm{~cm}$; brácteas del pedúnculo $15-22 \mathrm{~cm}$ largo, 5.5-7 cm ancho en la base, triangulares, a veces con algunos dientecillos en el margen, cartáceas, espina (2-)5-10 mm largo, brácteas de ramas primarias 8-10 cm largo, 4-5 cm ancho, deltoides. Flores (40-)60-80 por umbela, (5-)6-7 cm largo, ligeramente campanuladas, amarillas, con ovario verde; pedicelos 1-1.5 cm largo; 
ovario 2.5-3.5 cm largo, 5-7 $\mathrm{mm}$ ancho, triquetro a cilíndrico, cuello 3-5 mm largo, tubo 0.7-1(-1.2) cm diámetro, 0.7-1(-1.2) $\mathrm{cm}$ ancho en la porción distal; tépalos 1.5-2 cm largo, 3-5 mm ancho, los externos ligeramente más largos, involutos, a veces teñidos de púrpura, los internos aquillados, lineares, erectos, gruesos, ápice cuculado; filamentos 3-4.5 cm largo, insertados 1-2(-3) $\mathrm{mm}$ por debajo de la base de los tépalos, amarillos, anteras 1.5-2 cm largo, 2-3 $\mathrm{mm}$ ancho, versátiles, amari1las; estilo 4.5-6 cm largo, estigma trilobado. Cápsulas 4.5-5(-6.5) cm largo, 2-2.5 $\mathrm{cm}$ ancho, oblongas, cortamente estipitadas, rostro corto. Semillas 5-7 $\mathrm{mm}$ largo, 4-6 mm ancho, lunulares, sin ala, aplanadas, negras.

Tipo. Honduras, 8-12 km SE of Tegucigalpa along road to Zamorano, open rocky slope with pine, apr 1965, H.S. Gentry 20684 (neotipo, designado por Gentry (1982): US! isoneotipos: DES!, MEXU!).

Ejemplares examinados. México, OAXACA. Distrito Cuicatlán: Cerro del Cosahuico, San José El Chilar, C.A. Cruz Espinoza 491 (MEXU); Santiago Dominguillo, C.A. Cruz Espinoza 2037 (MEXU); Santiago Quiotepec, C.A. Cruz Espinoza 2144 (MEXU); $4 \mathrm{~km}$ al S de Tomellín camino a San Pedro Jaltepetongo, A. García-Mendoza et al. 7356 (MEXU); $6 \mathrm{~km}$ al SE de Tomellín camino a San Pedro Jaltepetongo, A. García-Mendoza et al. 7389 (MEXU); $16 \mathrm{~km}$ al SE de Dominguillo, por la carretera Tehuacán - Oaxaca, F. González-Medrano et al. 1747 (MEXU); Tomellín Canyon, J.N. Rose y J.S. Rose 11527 (US); Tomellín Canyon, W. Trelease s.n. (MO). Distrito Miahuatlán: $6 \mathrm{~km}$ al N de San Jerónimo Coatlán, A. García-Mendoza et al. 4594 (MEXU); $0.5 \mathrm{~km}$ al W de San Lorenzo Mixtepec, E. Hunn 500 (MEXU). Distrito Pochutla: Zacatal del Cerro Lobo, municipio San Miguel del Puerto, S. Salas et al. 5100 (MEXU, SERO); misma localidad, G. Salazar 7029 (MEXU). Distrito Sola de Vega: San Sebastián de Las Grutas, F. Palma C. 52 (MEXU). Distrito Tehuantepec: $2 \mathrm{~km}$ al N de La Concepción, $18 \mathrm{~km}$ al S de San Miguel Tenango, A. García-Mendoza y E. Martínez 3592, 3594 (MEXU); 5 $\mathrm{km}$ al S de San Miguel Tenango, A. García-Mendoza y E. Martínez 3599 (MEXU); $15 \mathrm{~km}$ al S de Buenos Aires camino a San Miguel Tenango, cultivada en el Jardín Botánico, A. García-Mendoza 6327, 6555 (MEXU); Ladera E del Cerro Guiengola, L. Torres C. et al. 344 (MEXU); El Ocotal, Cerro Guiengola, L. Torres C. et al. 944 (MEXU); Cerro El Gavilán, $10 \mathrm{~km}$ al E de Santiago Lachiguiri, J. Reyes $S$. 3577 (MEXU). Distrito Tlaxiaco: $3 \mathrm{~km}$ al S de Chalcatongo, camino a Yosondúa, A. García-Mendoza et al. 6837 (MEXU). Distrito Villa Alta: Cima del Cerro del Águila, $4 \mathrm{~km}$ al S de Santo Domingo Xagacia, A. García-Mendoza et al. 5770 
(MEXU); Santo Domingo Xagacia, F. Palma 74 (MEXU). Distrito Yautepec: Portillo de Nejapa, A. García-Mendoza 5691 (MEXU); Portillo de Nejapa, planta cultivada en el Jardín Botánico, A. García-Mendoza 6104 (MEXU); El Manguito, $6 \mathrm{~km}$ al E de El Camarón, A. García-Mendoza y H. Espinosa 7421 (MEXU); km 690 along highway to Tehuantepec, 10 miles SE of Camaron, H.S. Gentry 21886 (ARIZ, MEXU); 8 miles SE of Camaron along highway to Tehuantepec, H.S. Gentry y M. Gentry 23655 (ARIZ); between Tequisistlán and Totolapan, on road 195, $23 \mathrm{~km}$ NW of Río Hondo, B. Leuenberger y C. Schiers 2538 (MEXU); Subida a la torre de microondas San Cristóbal, $12 \mathrm{~km}$ al SE de El Camarón, R. Torres 12588 (MEXU).

Fenología. Florece de septiembre a diciembre y fructifica de diciembre a abril.

Hábitat y distribución. Agave seemanniana se desarrolla en laderas montañosas, sobre suelos arenosos derivados principalmente de rocas calizas, entre los 700-1700 m de altitud; forma parte de la selva baja caducifolia y de la ecotonía hacia el bosque subtropical de Quercus-Pinus. En Oaxaca se distribuye en la cuenca de los ríos Tomellín y Grande en el distrito de Cuicatlán y en las montañas de la Sierra Madre del Sur, principalmente en los distritos de Tlaxiaco, Miahuatlán, Pochutla, Yautepec y Tehuantepec.

Nombres comunes y usos. Bilía, chato, dób-bÉ, dua bla, tobalá, papalometl. Se utiliza para la elaboración de mezcal. En los distritos de Miahuatlán y Tlaxiaco también se emplea para preparar pulque (A. García-Mendoza et al. 4594, 6837 y E. Hunn 500).

Agave seemanniana se caracteriza por presentar rosetas mayores de un metro de alto, abiertas, con pocas hojas recurvadas, espatuladas a ampliamente lanceoladas, margen con mamilas prominentes y dientes con bases amplias y decurrentes. La inflorescencia posee ramas primarias, secundarias y ocasionalmente terciarias. Las flores se caracterizan por sus tubos cortos con los filamentos insertados 1-2(-3) mm por debajo de la base de los tépalos, los estambres son relativamente cortos, ya que sobrepasan al tubo por $1.5-2.5 \mathrm{~cm}$. Al igual que Agave potatorum, florece durante el otoño, con semillas viables durante el invierno y la primavera. Gentry (1982) menciona que habita desde Chiapas hasta Nicaragua; pero no obstante, que colectó plantas en Oaxaca, asignó los ejemplares a Agave potatorum (HSG 21886, 23655, 
véase también en su obra la fotografía inferior 17.21). Agave seemanniana coexiste con A. potatorum, A. angustifolia y A. convallis.

Los taxa tratados en este estudio, así como otras especies de Agave con las que coexisten, se pueden identificar con la siguiente clave:

1 Inflorescencia de apariencia espigada o racemosa

2 Inflorescencia espigada; flores en pares

3 Hojas 35-50 cm largo, 3.5-5.5 $\mathrm{cm}$ ancho, dientes $0.5-1 \mathrm{~cm}$ largo, espina 3-4.5 cm largo; flores $3.5-4 \mathrm{~cm}$ largo A. angustiarum

3 Hojas (40-)70-100 cm largo, (5.5-)8-12 cm ancho, dientes 1-2 cm largo, espina $4.5-5 \mathrm{~cm}$ largo; flores $3.5-5.5 \mathrm{~cm}$ largo ..................... A. convallis

2 Inflorescencia racemosa; flores en umbelas cortas, ramas de menos de $3 \mathrm{~cm}$ largo

4 Hojas lanceoladas, oblongas u ovadas, (5-)7-11 cm ancho, margen crenado, mamilado, dientes (5-)8-12 mm largo, retrorsos, antrorsos o rectos, espina 2-3(-4) cm largo A. nussaviorum subsp. nussaviorum

4 Hojas deltoides, (8-)14-20 cm ancho, margen córneo continuo, a veces con dientecillos de menos de de $5 \mathrm{~mm}$ largo, adpresos, espina (4-)6-10 cm largo A. nussaviorum subsp. deltoidea

1 Inflorescencia de apariencia paniculada

5 Hojas lineares o angostamente lanceoladas, 3.5-6 cm ancho, dientes 0.2-0.5 cm largo A. angustifolia

5 Hojas ampliamente lanceoladas, ovadas, oblongas o espatuladas, (5-)10-30(-40) $\mathrm{cm}$ ancho, dientes (0.4-)1-2 cm largo

6 Hojas 15-55 cm largo, (5-)8-22 cm ancho, ovadas, oblongas o espatuladas 7 Hojas 15-35 cm largo, (5-)8-10 cm ancho, erectas; ovadas, oblongas a lanceoladas; mamilas 4-7(-10) $\mathrm{mm}$ alto, dientes $0.4-0.6(-1.2) \mathrm{cm}$ largo, separados entre sí por $1-3 \mathrm{~cm}$ A. potatorum

7 Hojas 40-55 cm largo, (10-)14-22 cm ancho, recurvadas a erectas; espatuladas a ovadas; mamilas (0.7-)1-1.5(-2) cm alto; dientes (0.6-)1$1.5 \mathrm{~cm}$ largo, separados entre sí por (3-)4-5.5 cm A. seemanniana

6 Hojas 100-250 cm largo, 20-30(-40) cm ancho, ampliamente lanceoladas

8 Flores arregladas en grupos congestionados, esféricos; inflorescencia de apariencia oblonga; ramas primarias 20-30 cm largo; brácteas del pedúnculo cartáceas, prontamente deciduas A. atrovirens 
8 Flores arregladas en umbelas abiertas; inflorescencia de apariencia piramidal; ramas primarias $50-100 \mathrm{~cm}$ largo; brácteas del pedúnculo carnosas, persistentes A. salmiana

En las figuras 4 y 5 se muestran fotografías comparativas y en la figura 6 la distribución de los taxa tratados.
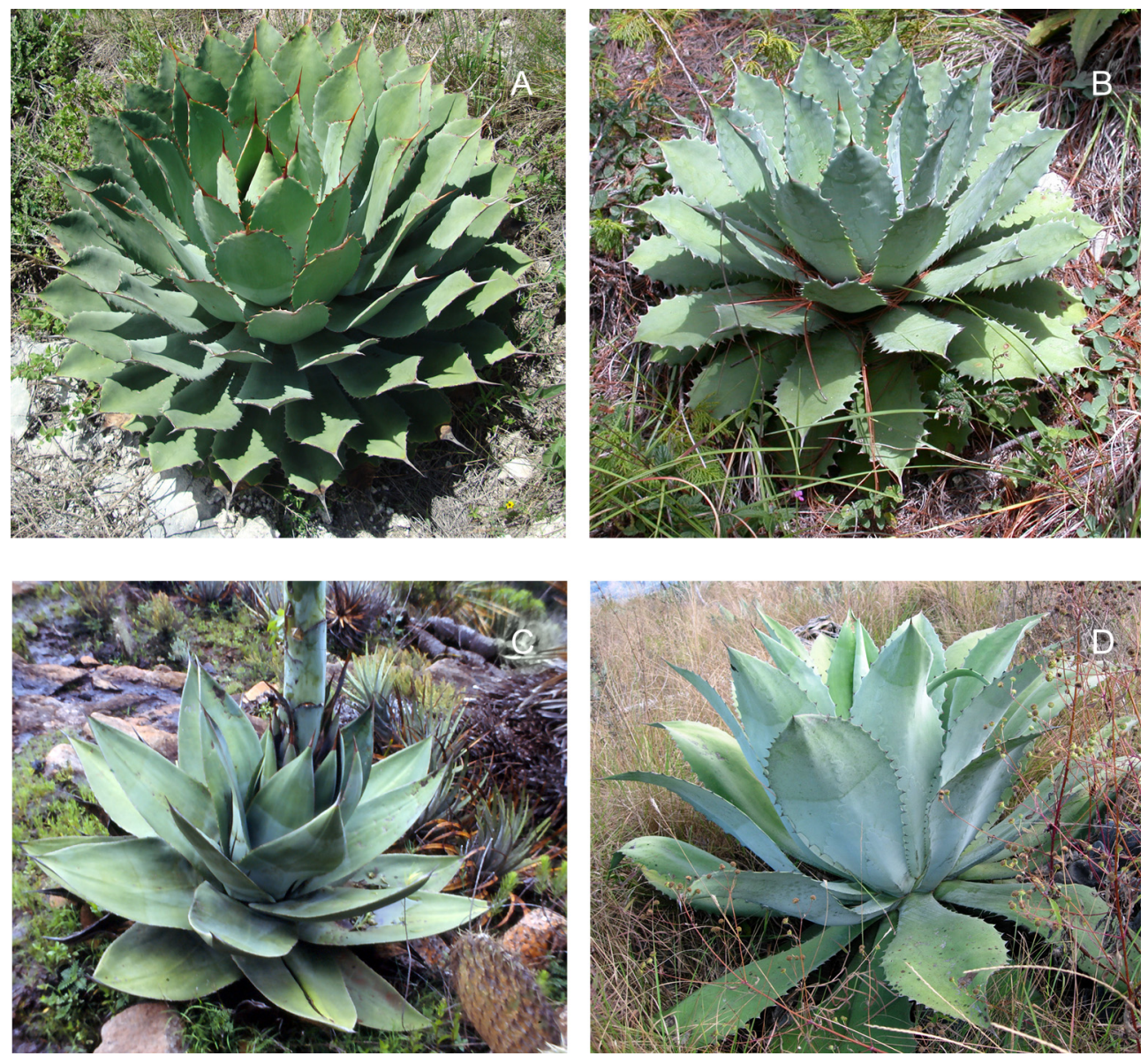

Fig. 4. Rosetas. A. Agave potatorum; B. Agave nussaviorum subsp. nussaviorum; C. Agave nussaviorum subsp. deltoidea; D. Agave seemanniana (foto G. Salazar). 
García-Mendoza: Revisión taxonómica del complejo Agave potatorum (Agavaceae)
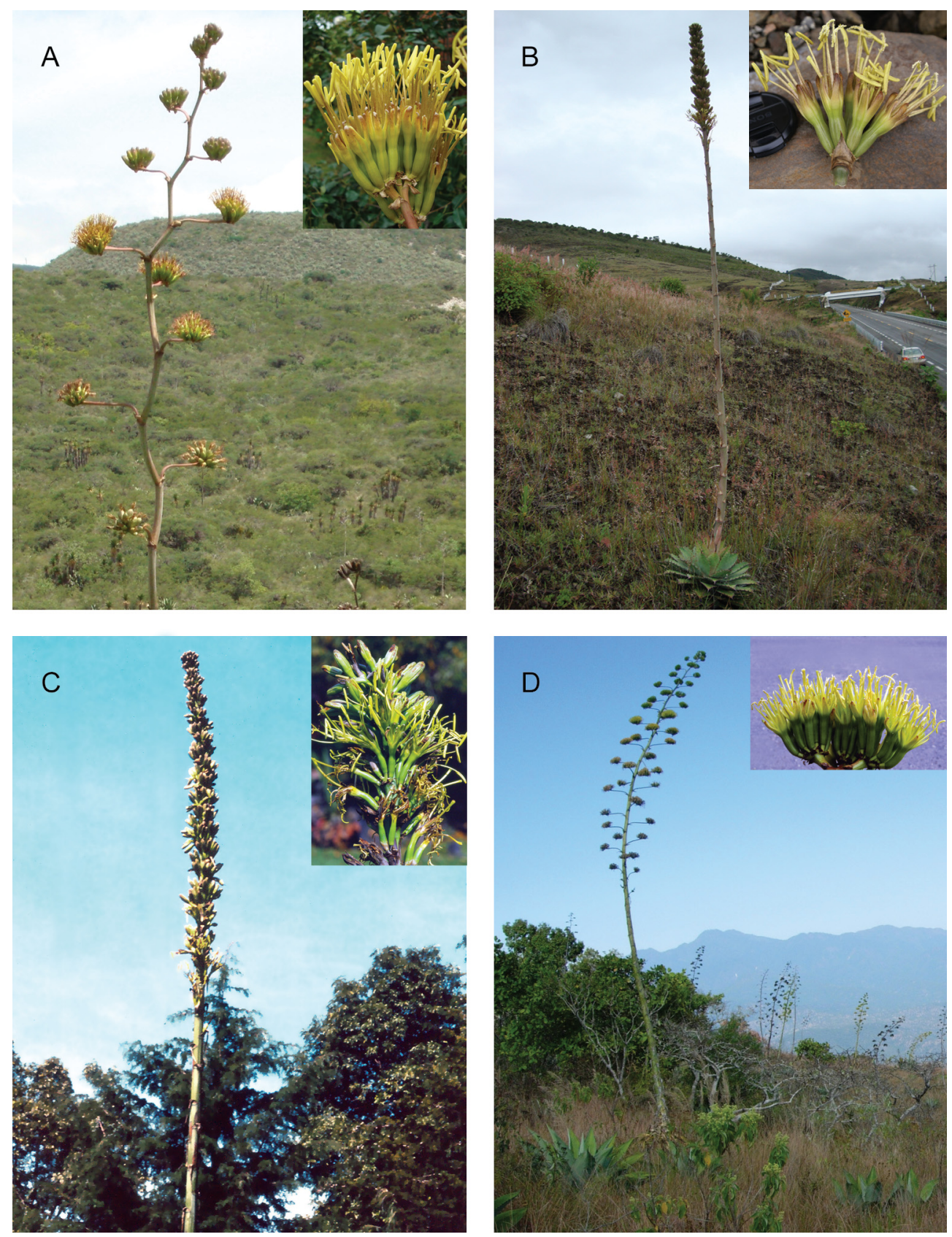

Fig. 5. Inflorescencias y flores. A. Agave potatorum; B. Agavenussaviorum subsp. nussaviorum; C. Agave nussaviorum subsp. deltoidea; D. Agave seemanniana (foto G. Salazar).. 


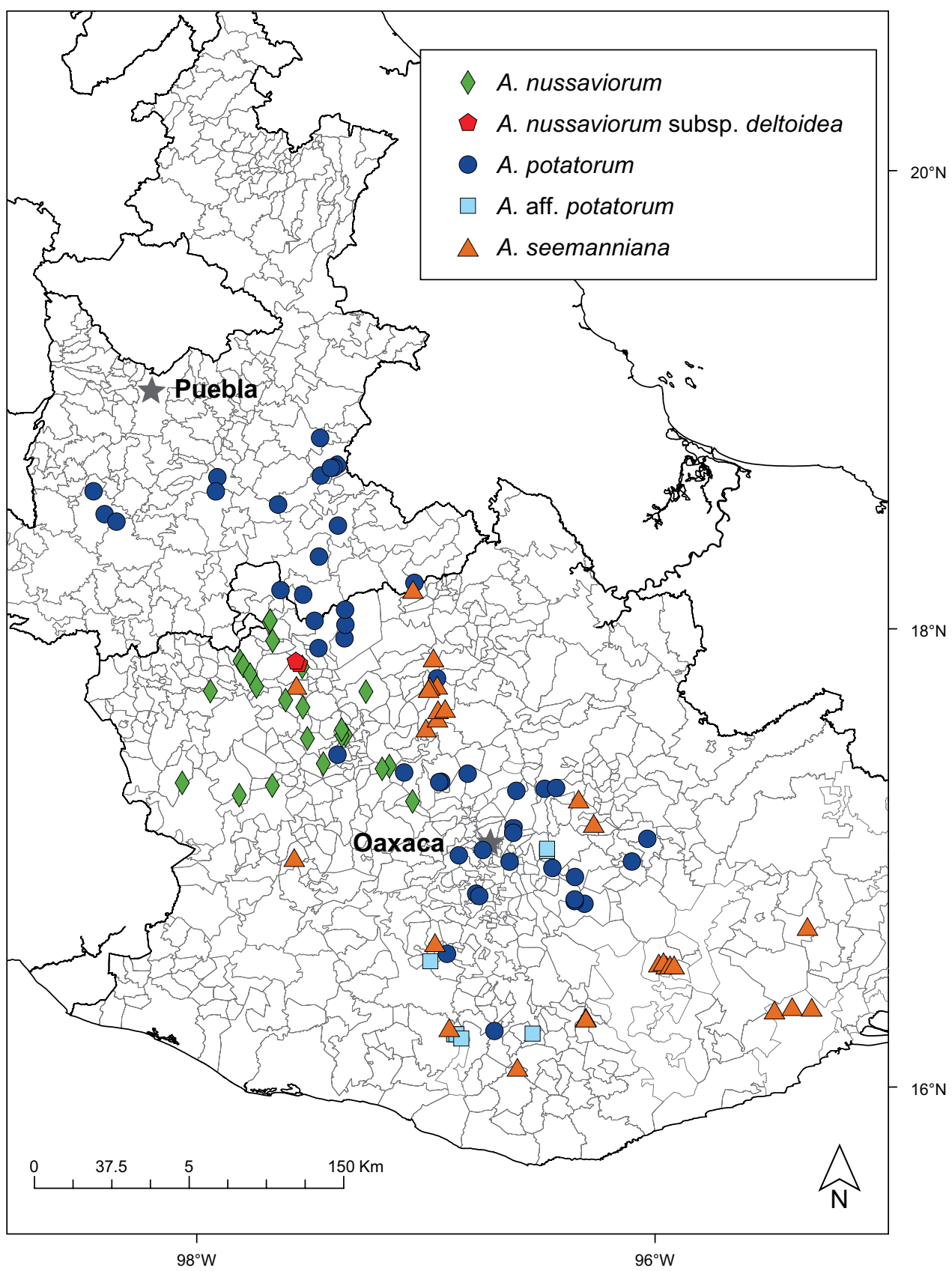

Fig. 6. Distribución de los taxa del complejo Agave potatorum en Oaxaca y Puebla. 


\section{AGRADECIMIENTOS}

Se agradece a los curadores de los herbarios ARIZ, DES, ENCB, MEXU, MO, OAX, SERO y US, por permitir consultar sus acervos. A los Drs. Fernando Chiang, Eloy Solano y Raquel Galván por la revisión crítica del manuscrito. Las diagnosis en latín fueron hechas por el primer revisor. Agradezco especialmente a Sonia Franco Martínez y Francisco Martínez Aguirre por su constante e invaluable colaboración en el trabajo de campo. Las figuras 1 y 2 son aportación de Elvia Esparza y la 3 de Albino Luna. Las fotos de Agave seemanniana son cortesía del Dr. Gerardo Salazar.

\section{LITERATURA CITADA}

Gentry, H. S. 1982. Agaves of continental North America. University of Arizona Press. Tucson, Arizona. $670 \mathrm{pp}$.

López, M. J. 2008. Estudio etnobotánico en el municipio de Santo Domingo Yodohino, Distrito de Huajuapan de León, Oaxaca. Tesis de licenciatura. Universidad Autónoma Chapingo. Chapingo, Estado de México. 220 pp.

Vásquez, G. 2006. Aprovechamiento integral del maguey pulquero (Agave salmiana) en Santa María Tlahuitoltepec Mixe Oaxaca. Tesis de licenciatura. Universidad Autónoma Chapingo. Chapingo, Estado de México. 103 pp.

Zuccarini, J. G. 1833. Über einige Pflanzen aus den Gattungen Agave und Fourcroya. Nova Acta Phys.-Med. Acad. Caes. Leop.-Carol. Nat. Cur. 16(2): 659-679, tab. 48-51. 